\title{
Reaction path for Te during surfactant-mediated epitaxial growth of GaAs (100)
}

\author{
C. D. Consorte, ${ }^{1}$ C. Y. Fong, ${ }^{1}$ M. D. Watson, ${ }^{1}$ L. H. Yang, ${ }^{2}$ and S. Ciraci ${ }^{3}$ \\ ${ }^{1}$ Department of Physics, University of California, Davis, California 95616 \\ ${ }^{2}$ Lawrence Livermore National Laboratory, Livermore, California 94551 \\ ${ }^{3}$ Department of Physics, Bilkent University, Bilkent Turkey \\ (Received 31 July 2000; published 9 January 2001)
}

\begin{abstract}
Using first-principles calculations and experimental evidence concerning the essential environment for surfactant-mediated epitaxial growth on the GaAs/Te(100) surface, we determine a short-ranged reaction path for the As $\leftrightarrow$ Te exchange that is energetically favorable and prepares the surface for continued layer-by-layer growth. Furthermore, we explain the required partial coverage of the surfactant atoms as well as the required presence of both As and $\mathrm{Ga}$ adatoms.
\end{abstract}

DOI: 10.1103/PhysRevB.63.041301

PACS number(s): 68.35.Fx, 71.15.-m

Since 1989, when Copel et al. ${ }^{1}$ demonstrated that the use of an additional surfactant species can dramatically affect the process of epitaxial growth, considerable experimental and theoretical effort has been put forth toward understanding the mechanism that allows the surfactant layer to float at the growth front. Experimental work has shown that the dominant effect of the surfactant is to restrict the epitaxial growth kinetics - reduction of the surface diffusion length (SDL) during homoepitaxial growth of elemental ${ }^{1,2}$ and III-V (Refs. 3 and 4) semiconductors as well as the heteroepitaxial growth of superlattices. ${ }^{5-8}$ The existence of an exchange process between the surfactant atoms and the growing species has been suggested by Grandjean and Massies (GM). ${ }^{4}$ By using first-principles calculations to determine the energy difference between the initial and final configurations, it has been shown that an exchange between surfactant dimers and growing species dimers is energetically favorable. ${ }^{9-11}$ These results were first obtained for the homoepitaxial growth of $\mathrm{Si}$ (Ref. 9) and later extended to the heteroepitaxial growth of GaAs/InAs. ${ }^{10,11}$ What remains to be determined is the actual reaction path that can lead the surfactant layer to an energetically favorable configuration at the growth front and allow the epitaxial growth process to perpetuate.

GaAs is an interesting material for this type of study because it has an added level of complexity over elemental semiconductors: there are two different growing species, hence the exchange process between growing atoms and surfactant atoms must be consistent with the zinc-blende lattice structure. Furthermore, because GaAs is an important material for fabrication of semiconductor devices, there is a considerable amount of experimental information available concerning the physical conditions under which the growth process can proceed. For example, it has been reported ${ }^{4}$ that the Te coverage must be less than one monolayer (ML), with $0.6 \pm 0.1$ ML being ideal. GM (Ref. 4) also reported that both the $\mathrm{Ga}$ and As growing species atoms (adatoms), again with fractional coverage (with As coverage as high as $0.7 \mathrm{ML}$ ), must be present in order for the Te surfactant atoms to segregate to the growth front. On the other hand, it has been determined that the dimerization of Te atoms is not energetically favorable. ${ }^{10,12}$ This information, plus the alternating species layers of any zinc-blende (100) surface, reduces the likelihood of a dimer exchange mechanism for surfactant- mediated epitaxial (SME) growth of GaAs (100). Unfortunately, the reconstruction of the surface used for SME growth of GaAs/Te(100) is still controversial. ${ }^{4,10,12}$ Without this crucial piece of information, a slab model for this type of study would cause further controversy.

In this paper, we report our results for the reaction path as determined from first-principles calculations. A cluster model simulating the $1 \times 1$ GaAs (100) surface in the neighborhood of a Te atom was built to incorporate the aforementioned experimental and theoretical evidence and to be independent of the surface reconstruction. Our objective was then to investigate the interaction between the Te surfactant atoms and the Ga and As adatoms that occurs on the Ga-terminated GaAs (100) surface during SME growth. In particular, we considered both the mechanism by which the exchange between the Te atom and the As adatom is initiated and the role played by the $\mathrm{Ga}$ adatom, thus necessitating its presence. In addition, it has been found within the context of SME growth, that partial coverage of the surfactant species is energetically favorable over full coverage. ${ }^{10}$ We will address the issue of why this is the case.

We perform first-principles electronic structure and totalenergy calculations based upon the local-density approximation $^{13}$ (LDA) of density-functional theory ${ }^{14}$ (DFT). We use the Ceperley-Alder ${ }^{15}$ exchange-correlation form as parametrized by Perdew and Zunger. ${ }^{16}$ The electronion interaction is treated using norm-conserving pseudopotentials ${ }^{17}$ and the wave functions are expanded in a plane-wave basis set. Because we are interested in accurately modeling the bonding between atoms, we include the frozen $d$ core states in the generation of the ionic pseudopotentials and set the kinetic energy cutoff of the plane-wave basis set to $25 \mathrm{Ry}$. This achieves a binding energy between atoms that is accurate to $1.0 \mathrm{mRy}(0.014 \mathrm{eV})$.

Because (a) the experimentally observed coverage of $\mathrm{Te}$ atoms is less than $1 \mathrm{ML}^{4}(\mathrm{~b})$ the exchange process happens very efficiently, i.e., it has an energy barrier of only $1.0 \mathrm{mRy}$ $(0.014 \mathrm{eV})$, and (c) the formation of Te dimers on the surface is unfavorable, ${ }^{10,12}$ the simplest model for studying the exchange process should involve only the atoms immediately around the surfactant atom. Since experimental ${ }^{4}$ and theoretical ${ }^{10,12}$ evidence suggest that the Te atom occupies the bridge site, we construct a cluster that simulates the Ga- 

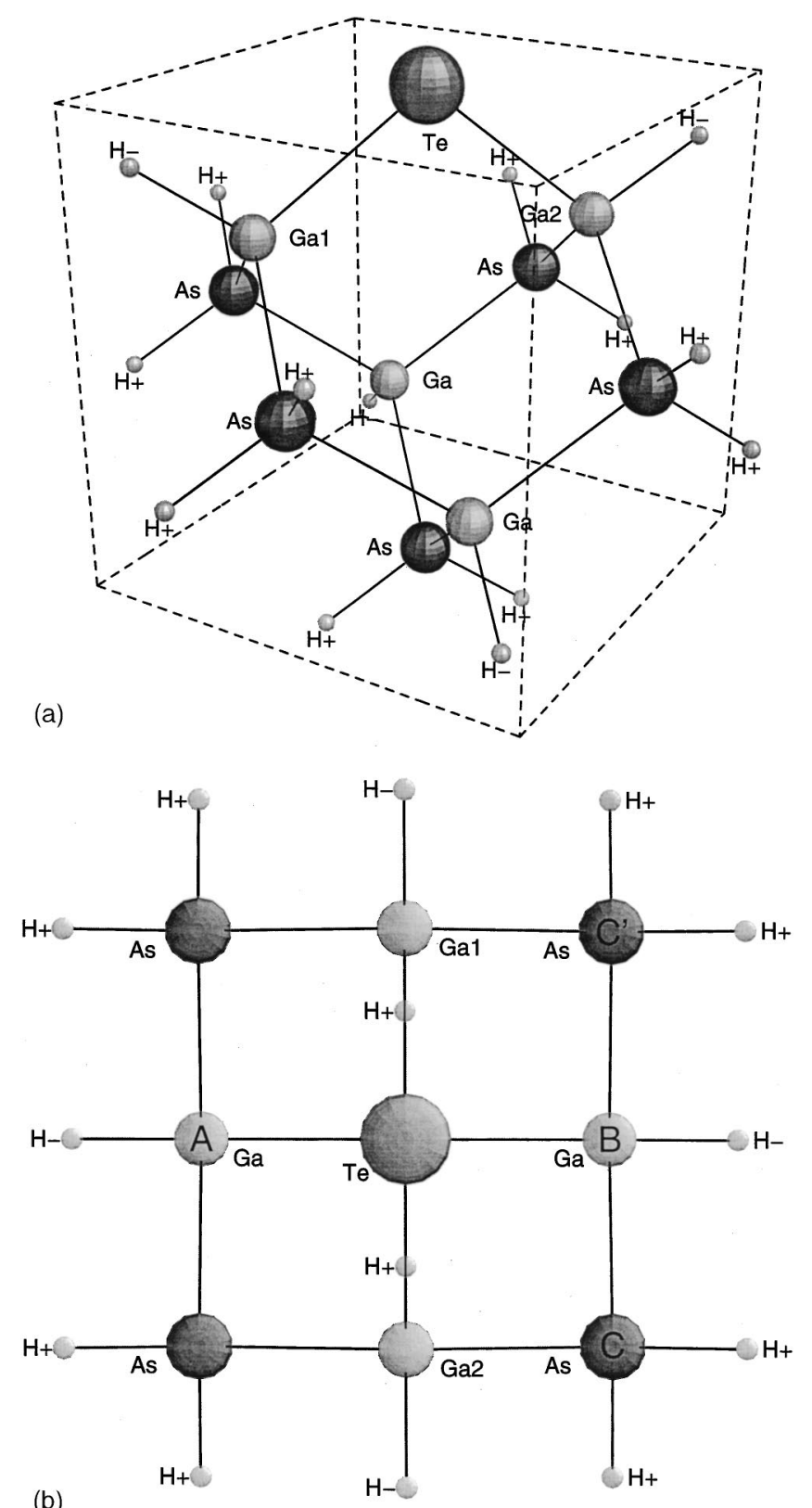

FIG. 1. (a) Cluster model in three-dimensional view. The dashed cube is used to guide the eyes to the location of the atoms. (b) Same model viewed from [001] direction. The largest sphere is Te, the dark spheres are As, and the light spheres are Ga. Open small spheres are $\mathrm{H}$.

terminated GaAs (100) surface in the neighborhood of a Te atom. To reduce the interactions between clusters, we place the cluster in a large $30 \times 30 \times 40 a_{B}^{3}(15.78 \times 15.78 \times 21.16$ $\AA^{3}$ ) unit supercell. The model is shown in Fig. 1(a), where the dashed box is not the unit cell but a guide to the eye. The top plane of the box defines the $1 \times 1$ surface unit cell centered at the Te atom. The cluster consists of 24 atoms: $4 \mathrm{Ga}$, $5 \mathrm{As}, 1 \mathrm{Te}$, and $14 \mathrm{H}$. The $\mathrm{Ga}$ and As atoms, representing the substrate, are arranged in the ideal zinc-blende structure with the Ga-As bond length of $4.583 a_{B}(2.424 \AA)$ determined from the bulk, which is in good agreement with the experimental value of $4.627 a_{B}(2.448 \AA) .{ }^{18}$ The $\mathrm{H}$ atoms, with

effective charges of $0.75 e$ and $1.25 e$, are used to saturate the dangling bonds, thus satisfying the electron counting rule for the substrate atoms, and making the bonding between those atoms more realistic.

We then added to the cluster model one of each type of growing species ( $\mathrm{Ga}$ and As adatoms), in accordance with experimental observation. We next considered the initial placement of these two adatoms. Although their presence is required, little is known about the details of their interaction with the surfactant atom, so the initial positions are based upon physical arguments which will be presented below. Experimental evidence shows that the As adatom must ultimately replace the $\mathrm{Te}$ atom at the bridge site. Because the Te-Ga bonds are planar [Te is bonded to $\mathrm{Ga}(1)$ and $\mathrm{Ga}(2)$ in Fig. 1(b)], moving the Te atom perpendicularly to that plane results in less strain on the bonds, and thus a smaller change in energy, than would occur for motion in the plane. Also, we expect that the Te atom will be repelled as the As adatom approaches, because the electronegativities of the two atoms discourage the formation of a bond between them. Finally, we note that at the surface, the As-Ga bond length of $4.583 a_{B}(2.424 \AA)$ is significantly smaller than the Te-Ga bond length which we determined to be $4.860 a_{B}(2.571 \AA$ ). Longer bonds generally imply weaker bonds and as an order of magnitude comparison, we found the bond energies of As-Ga and Te-Ga dimers to be $0.281 \mathrm{Ry}(3.822 \mathrm{eV})$ and $0.028 \mathrm{Ry}(0.381 \mathrm{eV})$, respectively. Thus, letting the As adatom approach the Te atom in the direction perpendicular to the Te-Ga bonds would allow the As adatom to get close enough to bind with the Ga substrate atoms while causing the minimum amount of strain to the Te-Ga bonds. So we initially place the As adatom at position $A$ in Fig. 1(b), which is above an empty metastable site at the surface as determined by Kley, Ruggerone, and Scheffler. ${ }^{19}$ We found that the qualitative features of the reaction path described below were insensitive to the vertical placement of the As adatom above the surface as long as it was between the initial Te height and the ideal height of that As layer. This provides a window of height that is $0.4 a_{B}(0.212 \AA)$ tall. It should also be noted that this position of the As atom is an interstitial site requiring a large coverage in order to guarantee its occupation. This is consistent with the experimental observation that the coverage of the As adatom is $0.7 \mathrm{ML}$.

In order for the $\mathrm{Ga}$ adatom to take part in the exchange process (which it must, otherwise its presence on the surface would not be required) we place it in the symmetrically similar metastable site on the other side of the Te atom, denoted by site $B$ in Fig. 1(b). When the As adatom eventually occupies the Te atom's initial position, it is reasonable to assume, given the zinc-blende structure, that it will also eventually bond with the $\mathrm{Ga}$ adatom. Thus we place the $\mathrm{Ga}$ adatom above the surface at a height that is near the ideal Ga height for the next layer of atoms in the crystal. Again, we find that the reaction path is insensitive to the exact location of the $\mathrm{Ga}$ adatom as long as it is above the initial height of the Te atom. If a reaction path can be found from this model, it will suggest that the exchange process is short-ranged, consistent with the reduction of the SDL, and will support the statement ${ }^{4}$ that it happens very efficiently. 


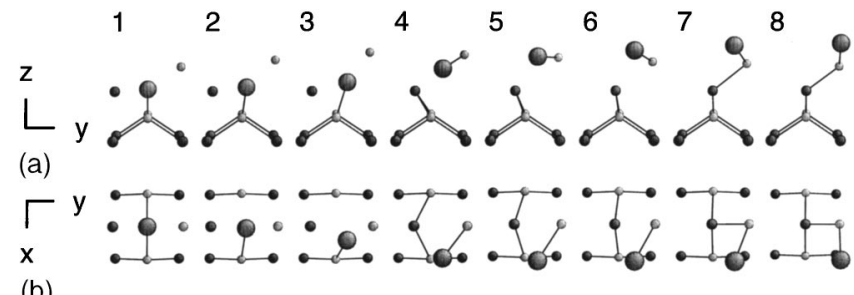

(b)

FIG. 2. A snapshot series of a pathway for the exchange process between the surfactant, Te, and the As adatom with the presence of a Ga adatom. (a) Side view, (b) top view. Large sphere is the Te, the dark small sphere is the As adatom, and the light small sphere is the $\mathrm{Ga}$ adatom. For clarity, most of the substrate atoms have been omitted.

We determined the reaction path by performing a series of total-energy calculations on our cluster model. For each calculation, we fixed the 23 atoms that model the substrate, since relaxation of the substrate will definitely reduce the total energy, and allowed only the Te and the two adatoms to move. A move was not accepted if the increase in the total energy of the system was greater than $1.0 \mathrm{mRy}(0.014 \mathrm{eV})$, which is the accuracy of our calculations. A variation of this scheme has been used by others to determine the energy barriers of proposed pathways. ${ }^{20}$ Because we are using a model, we imposed restrictions on the movements of the adatoms to reduce the complexity (degrees of freedom). Since it is anticipated that the As adatom will replace the Te atom at the bridge site, we require that the As adatom move there directly from site $A$ [Fig. 1(b)] without any lateral displacement. Furthermore, we do not allow the Te atom or the As adatom to retrace their steps. Another factor to consider is that experimentally, SME growth usually occurs at around $600{ }^{\circ} \mathrm{C}$ whereas DFT-LDA calculations simulate $0 \mathrm{~K}$. Raising the temperature adds 0.006 Ry $(0.082 \mathrm{eV})$ of kinetic energy to the system. While this is not enough energy to spontaneously break the Te-Ga bonds, it can certainly help facilitate the Te↔As exchange. In addition, this temperature effect would certainly allow the Ga adatom to move around in the neighborhood of its initial position. However, we restrict it to vertical displacements only. By removing these degrees of freedom from the system, we in effect adopt a worst-case scenario for the reaction path.

Figure 2 shows the side and top views for consecutive stages in the reaction path described below. We initiate the movements of the Te atom and the As adatom along the $+\mathbf{y}$ direction [perpendicular to the plane containing $\mathrm{Te}, \mathrm{Ga}(1)$, and $\mathrm{Ga}(2)]$ as described in the previous section. The total energy is not increased until the Te atom has moved $0.6 a_{B}$ $(0.032 \AA)$ and the As adatom $0.05 a_{B}(0.026 \AA)$, with the Ga adatom fixed. The increase in the energy is $1.0 \mathrm{mRy}(0.014$ $\mathrm{eV}$ ). If we allow the $\mathrm{Ga}$ adatom to move, the energy will decrease. Our restrictions allow it to move only vertically. Since there is no atom above the Ga adatom, and any arbitrary vertical displacement of the Ga adatom would reduce the total energy, we now hold the distance between the Te atom and the $\mathrm{Ga}$ adatom fixed at $4.46 a_{B}(2.359 \AA)$. When the displacements of the As and Te atoms are increased to 0.2 and $0.15 a_{B}$ (0.106 and $\left.0.079 \AA\right)$ in the $\mathbf{y}$ direction, respec- tively, and the $\mathrm{Ga}$ adatom has moved upward accordingly, the total energy is reduced by $0.038 \mathrm{Ry}(0.517 \mathrm{eV})$. This demonstrates that if the $\mathrm{Ga}$ adatom is allowed to move, the initial barrier of $1.0 \mathrm{mRy}(0.014 \mathrm{eV})$ can be avoided. In order for the exchange process to continue, the Te atom should move above and to either the right or the left side of the $\mathrm{Ga}$ adatom (along the $\mathbf{x}$ direction). The final position of the Te atom will be the next bridge site in the next As layer indicated by site $C$ or $C^{\prime}$ (cf. Fig. 1). The availability of this position requires that the coverage of Te be less than $1 \mathrm{ML}$ as indicated in the experiments, otherwise the movement will be hindered by the strong repulsion of the nearby Te atoms. To proceed to its final position, we first move the Te atom laterally (in the $+\mathbf{x}$ direction as depicted in frames 3 and 4 of Fig. 2). The energy steadily decreases as the As adatom simultaneously approaches its final site. When the Te atom reaches its final position in the $\mathbf{x}$ direction (frame 5 in Fig. $2)$, there is a reduction in energy of $0.05 \mathrm{Ry}(0.68 \mathrm{eV})$. At this point, the Ga adatom is higher than the Te atom. We can then increase the $\mathbf{z}$ component of the Te atom's position while simultaneously lowering the Ga adatom (frames 5-8 in Fig. 2) without encountering an energy barrier. Now the Te atom and the two adatoms are in the appropriate positions on the surface to allow the SME growth process to continue. If the Te atom were forced upward initially, it would ultimately occupy a void region on the Ga layer which would prevent further progress of the SME growth process. Furthermore, the Te and Ga bonds would be stretched, which would cost energy. So it is clear that the As $\leftrightarrow$ Te exchange is not vertical in nature. In addition, we have determined that the role of the $\mathrm{Ga}$ adatom is to prevent the Te atom from diffusing away due to the energy gained from the bonding of the As adatom with $\mathrm{Ga}(1)$ and $\mathrm{Ga}(2)$, and to guide the Te atom into its next bridge site.

In summary, although both experimental and theoretical studies have determined the necessary conditions for SME growth to occur on the $\mathrm{GaAs} / \mathrm{Te}(100)$ surface, little was known about the actual mechanism that initiates the exchange between the surfactant atoms and the growing species atoms. Furthermore, what speculation there has been has only considered the energetics of a direct exchange. However, for a zinc-blende structure any direct exchange, be it atomic or dimeric in nature, would result in a site mismatch for the surfactant. Such a process also costs energy due to stretching of the Te-Ga bonds. Based upon the experimental evidence about the presence and coverage of the Te atoms, As and Ga adatoms, as well as the established observation that SME growth results in a reduced SDL of the growing species, we have constructed a cluster model that represents a $1 \times 1$ structure of the $\mathrm{GaAs} / \mathrm{Te}(100)$ surface in the neighborhood of the Te atom. With first-principles total-energy calculations and physically motivated restrictions on atomic movement, we determined a reaction path that is energetically favorable (essentially no barrier) and is short ranged. Our results show (1) how the Te surfactant atoms segregate to the growth front in such a way that they can occupy new As substitutional sites and can thus perpetuate the layer-bylayer growth, (2) why Ga adatoms are needed during the 
experiments, and (3) that the exchange process is very efficient, i.e., short-ranged with no energy barrier more than our calculational accuracy.

A crucial point is that the Te atom must occupy a bridge site. Moreover, the Te atom must be more weakly bound to the surface and have longer bonds than the As adatom that replaces it. Finally, because the Te atom is larger than the substrate atoms, it was able to segregate to the growth front rather than diffusing into the surface. These three factors all work together to initiate, then perpetuate the incorporation of the As and Ga adatoms into the surface and the segregation of the Te atom to the growth front. Thus, these factors might be considered as criteria for selecting a surfactant for a particular surface.

This work was supported in part by the National Science Foundation under Grant No. INT-9872053 and the Campus Laboratory Collaboration Grant of the University of California. L.H.Y. is also supported by the DOE.
${ }^{1}$ M. Copel, M. C. Reuter, Efthimios Kaxiras, and R. M. Tromp, Phys. Rev. Lett. 63, 632 (1989).

${ }^{2}$ R. M. Tromp and M. C. Reuter, Phys. Rev. Lett. 68, 954 (1991).

${ }^{3}$ J. Massies and N. Grandjean, Phys. Rev. B 48, 8502 (1993).

${ }^{4}$ N. Grandjean and J. Massies, Phys. Rev. B 53, R13 231 (1996).

${ }^{5}$ N. Grandjean, J. Massies, and V. H. Etgens, Phys. Rev. Lett. 69, 796 (1992).

${ }^{6}$ N. Grandjean, J. Massies, C. Delamarre, L. P. Wang, A. Dubon, and J. Y. Laval, Appl. Phys. Lett. 63, 66 (1993).

${ }^{7}$ Bert Voigtländer, Andre Zinner, Thomas Weber, and Hans P. Bonzel, Phys. Rev. B 51, 7583 (1995).

${ }^{8}$ S. Schintke, U. Resch-Esser, N. Esser, A. Krost, W. Richter, and B. O. Fimland, Surf. Sci. 377-379, 953 (1997).

${ }^{9}$ Takahisa Ohno, Phys. Rev. Lett. 73, 460 (1994).

${ }^{10}$ R. H. Miwa, A. C. Ferraz, W. N. Rodrigues, and H. Chachum, Surf. Sci. 415, 20 (1998).
${ }^{11}$ R. H. Miwa and A. C. Ferraz, Appl. Surf. Sci. 123-124, 449 (1998).

${ }^{12}$ S. Gundel and W. Faschinger, Phys. Rev. B 59, 5602 (1999).

${ }^{13}$ W. Kohn and L. J. Sham, Phys. Rev. 140, A1133 (1965).

${ }^{14}$ P. Hohenberg and W. Kohn, Phys. Rev. 136, B864 (1964).

${ }^{15}$ D. M. Ceperley and B. I. Alder, Phys. Rev. Lett. 45, 566 (1980).

${ }^{16}$ J. P. Perdew and A. Zunger, Phys. Rev. B 23, 5048 (1981).

${ }^{17}$ L. Kleinman and D. M. Bylander, Phys. Rev. B 46, 16067 (1992).

${ }^{18}$ R. W. G. Wyckoff, Crystal Structure (Interscience, New York, 1963), Vol. 1, p. 110.

${ }^{19}$ A. Kley, P. Ruggerone, and M. Scheffler, Phys. Rev. Lett. 79, 5278 (1997).

${ }^{20}$ C. W. Oh, E. Kim, and Y. H. Lee, Phys. Rev. Lett. 76, 776 (1996). 